\title{
Avanço antrópico na bacia hidrográfica do Rio Barigui entre 1999 e 2019 e seu impacto sobre o escoamento superficial avaliado pela alteração da sua curva-número $(\mathrm{CN})$
}

\author{
Anthropic expansion in the Barigui River watershed \\ between 1999 and 2019 and its impact on surface flow \\ assessed by the change of its curve-number (CN)
}

Juliana Leithold ${ }^{1}$, Marcio Augusto Reolon Schmidt² (D), Cristóvão Vicente Scapulatempo Fernandes ${ }^{1}$ (i)

${ }^{1}$ Universidade Federal do Paraná - UFPR, Curitiba, PR, Brasil. E-mails: juleithold@gmail.com, cris.dhs@ufpr.br 2Universidade Federal de Uberlândia - UFU, Uberlândia, MG, Brasil. E-mail: marcio.schmidt@ufu.br

Como citar: Leithold, J., Schmidt, M. A. R. \& Fernandes, C. V. S. (2020). Avanço antrópico na bacia hidrográfica do Rio Barigui entre 1999 e 2019 e seu impacto sobre o escoamento superficial avaliado pela alteração da sua curva-número (CN). Revista de Gestão de Água da América Latina, 17, e25. https://doi.org/10.21168/rega.v17e25.

\begin{abstract}
RESUMO: 0 acompanhamento do avanço antrópico em bacias hidrográficas a partir da avaliação da cobertura do solo tem grande utilidade na gestão de recursos hídricos, como no caso do modelo chuva-vazão CN-SCS que utiliza o conceito de curva-número $(\mathrm{CN})$ para estimar qual o potencial da precipitação tornar-se escoamento superficial com base na cobertura e tipo do solo em uma bacia hidrográfica. Nesta pesquisa foi avaliada a evolução da cobertura do solo na bacia do Rio Barigüi entre os anos de 1999 e 2019 e seu impacto sobre as características hidrológicas com base na variação da CN. Para tanto, imagens Landsat foram classificadas para os anos em estudo e os tipos hidrológicos de solo na bacia definidos. Os resultados indicaram que houve relevante avanço antrópico na região, com aumento de urbanização $\left(\sim 21 \mathrm{~km}^{2}\right)$ e redução de áreas florestadas $\left(\sim 22 \mathrm{~km}^{2}\right)$. No entanto, tal avanço não afetou de forma expressiva o valor de $\mathrm{CN}$ total da bacia, devido ao tipo do solo predominante, mais impermeável, e à forma em que ocorreram as alterações na cobertura do solo, com aterro de áreas alagadiças e consequente acréscimo de permeabilidade em algumas regiões.
\end{abstract}

Palavras-chave: CN-SCS; Landsat; Cobertura do Solo; Sensoriamento Remoto.

ABSTRACT: The monitoring of anthropic expansion in river basins from the assessment of land cover is very useful for water resources management, as in the case of the CN-SCS rainfall-runoff model, which uses the concept of curve number $(\mathrm{CN})$ to estimate which the rainfall potential to become runoff based on the watershed's soil and land cover. This research evaluated the evolution of land cover in the Barigüi River basin between 1999 and 2019 and its impact on hydrological characteristics based on the variation of their CN. Therefore, Landsat images were classified for the study years and the hydrological types of soil in the basin were defined. The results indicated that there was a significant anthropic advance in the region, with an increase in urbanization $\left(\sim 21 \mathrm{~km}^{2}\right)$ and a reduction in forested areas $\left(\sim 22 \mathrm{~km}^{2}\right)$. However, this advance did not affect significantly the total $\mathrm{CN}$ value of the basin, due to the predominant, more impermeable type of soil, and how the changes in land cover occurred, with flooding of wetlands and the consequent increase in permeability in some regions.

Keywords: CN-SCS; Landsat; Land Cover; Remote Sensing.

\section{INTRODUÇÃO}

A água é um recurso essencial à humanidade e ao meio ambiente, e sua disponibilidade em quantidade e qualidade adequadas é foco de muita atenção tanto no passado, quanto na atualidade. No entanto, tais características são diretamente afetadas pelas condições de ocupação das bacias

Recebido: Novembro 09, 2020. Revisado: Dezembro 07, 2020. Aceito: Dezembro 09, 2020. 
hidrográficas, e a avaliação das características de cobertura do solo proporciona informações dos impactos ambientais a que os recursos hídricos estão suscetíveis. Bacias hidrográficas submetidas a forte ação antrópica possuem maior impermeabilização do solo, com rápido escoamento de águas pluviais, levando a problemas com inundações e enchentes em áreas mais sensíveis (Tong \& Chen, 2002; Paule-Mercado et al., 2018). Desta forma, o monitoramento do avanço antrópico permite nortear a gestão de recursos hídricos, auxiliando o planejamento de ações emergenciais ou corretivas de curto a longo prazo.

A utilização de técnicas baseadas em sistemas de informação geográfica (SIG) contribui de forma significativa na avaliação das alterações e das características de uma bacia hidrográfica. SIGs são ferramentas que possuem como objetivo armazenar, analisar e integrar diversas informações espaciais, com aplicação em diversas áreas do conhecimento. Na gestão de recursos hídricos possibilitam a agregação de diferentes parâmetros como dados de precipitação, tipo e cobertura do solo, a partir de, por exemplo, sobreposição de mapas temáticos (Ramakrishnan et al., 2009).

Dentre suas ferramentas está o sensoriamento remoto que tem como objetivo o estudo dos fenômenos e interações que ocorrem na superfície terrestre, porém sem contato direto, realizado a partir da interação dos seus componentes com a radiação eletromagnética pelo uso de aeronaves, espaçonaves, sensores, equipamentos de transmissão e processamento de dados (Gonçalves et al., 2015; Vale et al., 2018).

Diferentes sensores fornecem variados tipos de imagens, com disponibilidade de resolução espacial, temporal, espectral e radiométrica, além de custos, variados (Vale et al., 2018). Assim, a escolha do tipo de imagem utilizada irá depender dos objetivos e necessidades do estudo. 0 uso de imagens Landsat muitas vezes acaba por ser o mais interessante devido à disponibilidade de uma maior série temporal de imagens (a série Landsat teve início ainda na década de 1970), além da disponibilização de imagens de forma gratuita. Possui resolução espacial de $30 \mathrm{~m}$ para as bandas espectrais azul (B), verde $(G)$, vermelho $(R)$ e infravermelhos próximo ( $\left.I_{n}\right)$ e médio $\left(I_{m}\right)$ (Centeno, 2009).

Para determinar a forma de ocupação de uma bacia hidrográfica é possível utilizar-se de imagens de satélite a partir de diferentes composições entre suas bandas espectrais. Cada composição destaca características diversas da superfície terrestre, como maior ou menor umidade do solo, conforme sua capacidade de reflexão da energia eletromagnética solar, permitindo a diferenciação entre as formas de cobertura do solo, como vegetação (densa ou rasteira natural e agrícola), construções, solo exposto, entre outras, permitindo a classificação da cobertura do solo de forma tanto supervisionada por um técnico, como não supervisionada com uso de padrões reconhecidos por programas de computador (Centeno, 2009; Mendes \& Cirilo, 2013).

Ainda, a partir de cálculos entre as intensidades de cada pixel em cada banda da imagem é possível determinar índices radiométricos que podem auxiliar esta classificação, como o NDVI (Normalized Difference Vegetation Index), o NDWI (Normalized Difference Water Index) e o NDBI (Normalized Difference Built-Up Index), que indicam a presença de vegetação, áreas úmidas e construções, respectivamente (Lee et al., 2010; Moreira Filho \& Tavares Junior, 2016).

Um exemplo de aplicação direta de um SIG na gestão de recursos hídricos é a partir do uso de modelos hidrológicos que se utilizam do uso e cobertura dos solos, como é o caso do modelo empírico curva-número do Soil Conservation Service (CN-SCS). Este se baseia nas características do solo e na forma de ocupação de uma bacia hidrográfica para estimar a faixa de permeabilidade da bacia e, por consequência, a capacidade da transformação da chuva em vazão (United States Department of Agriculture, 1986; Mishra \& Singh, 1999; Mishra et al., 2003).

Este método é mais bem aplicado para pequenas bacias, com área inferior a $250 \mathrm{~km}^{2}$, e pode ser utilizado para avaliar a evolução da ocupação do solo e sua interferência sobre os processos hidrológicos, porém tem sido empregado também em bacias de maior porte a partir da subdivisão em sub-bacias (United States Department of Agriculture, 1986). Apesar de sua simplicidade, o método SCS-CN permanece sendo utilizado em diversos estudos recentes (Bartlett et al., 2016; Nachshon et al., 2016; Beck et al., 2017; Kayet et al., 2018; Gaglio et al., 2019; Walega et al., 2020).

Neste sentido, em bacias com forte ação antrópica, como a do Rio Barigüi, afluente da margem direita do Rio Iguaçu, na Região Metropolitana de Curitiba, Paraná, com área de aproximadamente $267 \mathrm{~km}^{2}$, e que possui grande complexidade em sua ocupação (Superintendência de Desenvolvimento de Recursos Hídricos e Saneamento Ambiental, 2002; Machado et al., 2014) pode ter a evolução do seu uso do solo avaliada de acordo com dados de sensoriamento remoto e pelo método da curva-número, a fim de verificar a alteração de suas características hidrológicas nos últimos anos. Complementarmente, um estudo semelhante, porém na Bacia do Ivaí, também no Estado do Paraná, 
foi realizado por Soares et al. (2017) que avaliaram a evolução da cobertura do solo ao longo do período 1986 a 2011 e a consequente alteração na CN da bacia hidrográfica. No entanto, devido à grande extensão da bacia (área de estudo superior a $34 \mathrm{mil} \mathrm{km}^{2}$ ), esta foi dividida em 14 sub-bacias a as alterações da CN foram avaliadas pixel a pixel.

No que concerne à definição de ferramentas para se definir os instrumentos de gestão de recursos hídricos, uma abordagem que permita estimar a dinâmica de uso da terra faz-se ainda necessária, de forma a permitir integrar a base de informações quali-quantitativa, permitindo uma melhor abordagem pelos gestores. Neste contexto, ao poder estimar adequadamente as transições temporais de uso da terra para a adequada representação hidrológica, obtida através das estimativas de alterações do $\mathrm{CN}$, buscou-se, com esta pesquisa responder às seguintes questões: i) é possível avaliar a evolução do uso e ocupação do uso do solo na Bacia Hidrográfica do Rio Barigüi entre os anos 1999 e 2019 através da classificação supervisionada de imagens Landsat e da determinação de índices radiométricos? e; ii) a variação da capacidade de escoamento superficial da bacia pode ser obtido a partir da variação da CN da bacia neste período?

\section{MATERIAL E MÉTODOS}

\section{Área de estudo}

A Bacia do Rio Barigüi está localizada no Estado do Paraná, na região Sul do Brasil, abrangendo os municípios de Almirante Tamandaré, onde fica sua nascente, Araucária, Colombo e Curitiba, conforme Figura 1. Seu rio principal é afluente da margem direita do Rio Iguaçu e tem extensão de aproximadamente $66 \mathrm{~km}$, com uma bacia de drenagem com área de cerca de $267 \mathrm{~km}^{2}$ e vazão média em sua foz de 4,8 $\mathrm{m}^{3} / \mathrm{s}$ (Superintendência de Desenvolvimento de Recursos Hídricos e Saneamento Ambiental, 2002; Instituto Ambiental do Paraná, 2018).

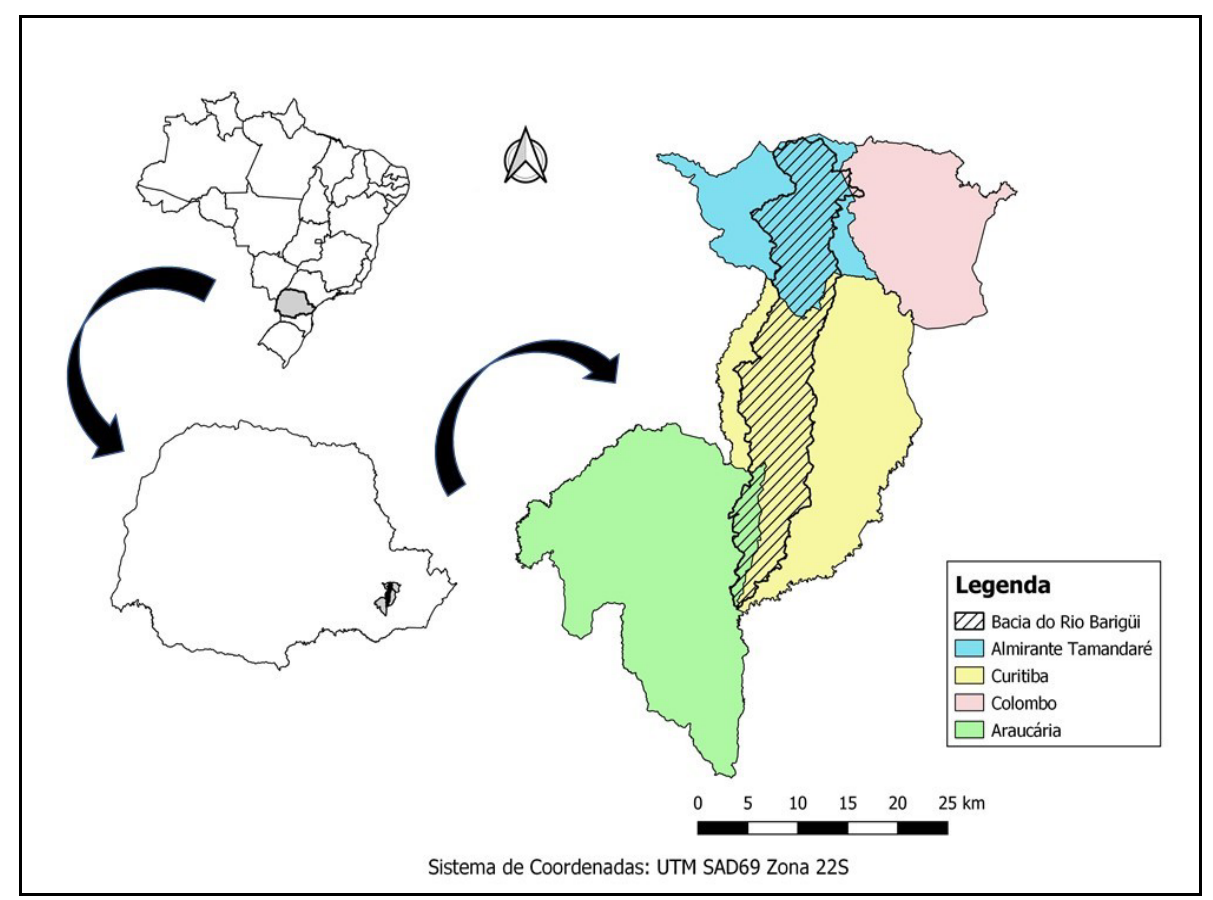

Figura 1 - Localização da Bacia Hidrográfica do Rio Barigüi em relação ao Brasil e ao Estado do Paraná Fonte: Elaborado a partir de dados de Ambdata (2019) e Instituto de Terras, Cartografia e Geologia do Paraná (2019)

A Figura 2 apresenta as características topográficas e hidrológicas da Bacia do Rio Barigüi com base no Modelo Digital de Elevação (MDE), obtido a partir do projeto Topodata do Instituto Nacional de Pesquisas Espaciais (INPE), e no recorte da bacia do Rio Barigüi e sua hidrologia, disponível no site do Instituto das Águas do Paraná (Instituto Água e Terra, 2000). 


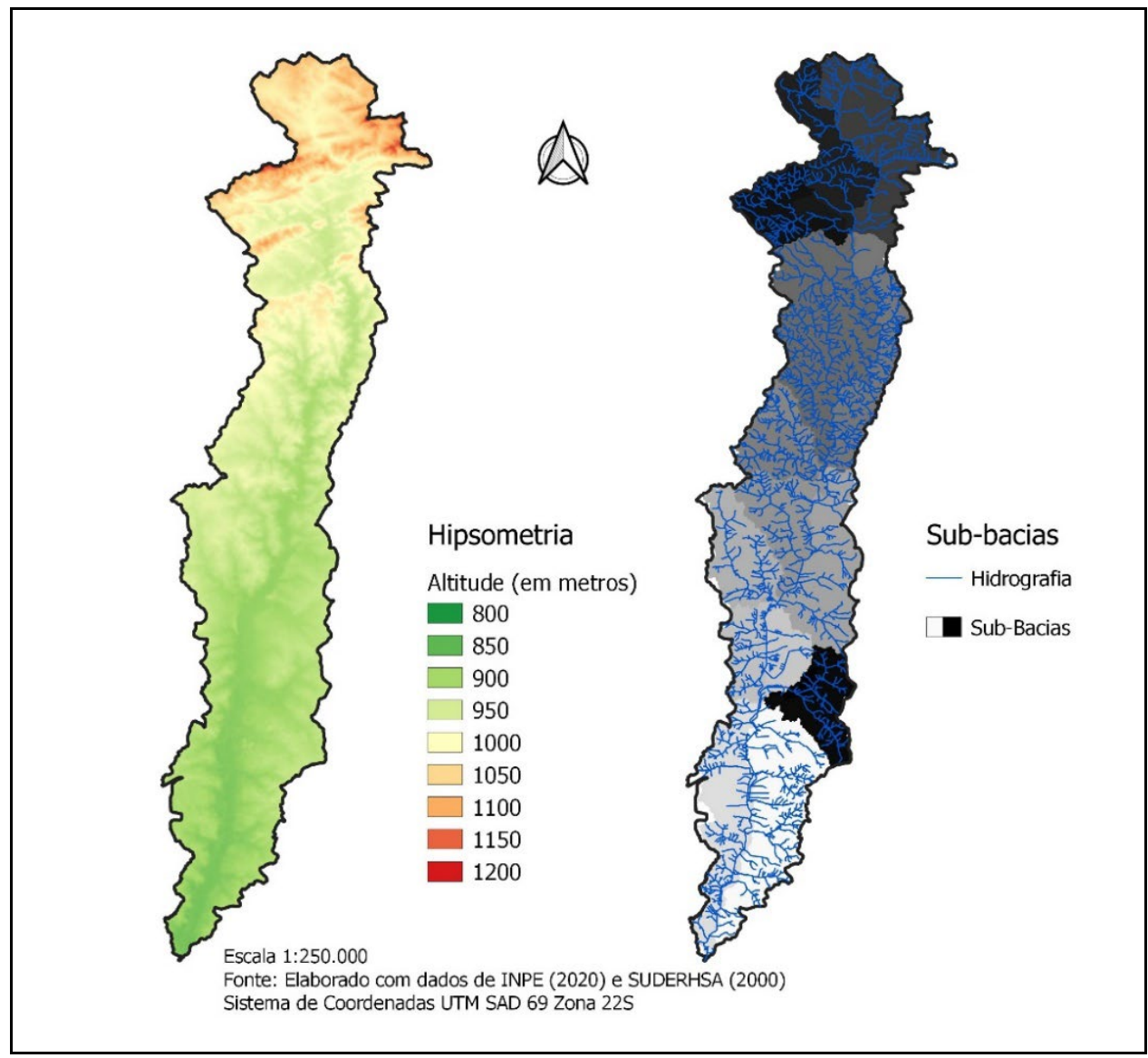

Figura 2 - Hipsometria, sub-bacias e hidrografia da Bacia do Rio Barigüi

A ocupação no solo da bacia é bastante diversificada, sendo sua região de montante, mais ao norte, essencialmente rural e com áreas de vegetação densa, contando com pequenos núcleos urbanos mais dispersos. Por outro lado, a região central da bacia tem alta densidade urbana, com edificações residenciais uni e multifamiliares, comerciais e de serviço. Na região mais a jusante, ao sul da bacia, há forte presença de indústrias, visto que nela se localiza a Cidade Industrial de Curitiba (CIC) e o setor industrial de Araucária. Tais características, em conjunto ao formato alongado da bacia e padrão de drenagem dendrítico favorecem o rápido escoamento na bacia, tornando as regiões mais próximas ao rio bastante propensas a enchentes (Froehner \& Martins, 2008; Instituto Ambiental do Paraná,, 2018).

\section{Classificação da cobertura do solo}

As imagens de satélite, a partir das diferenças na absorção, reflectância e transmitância de energia nas diferentes bandas do espectro eletromagnético de acordo com variadas coberturas do solo, geram informações que, analisadas através de ferramentas SIG permitem, por exemplo, a avaliação da cobertura do solo de bacias hidrográficas (Centeno, 2009; Mendes \& Cirilo, 2013).

Além da composição de imagens a partir das bandas disponíveis (visível, infravermelho próximo, médio e termal), é possível também obter índices radiométricos de cobertura com base nas diferenças normalizadas entre a reflectância das bandas. Alguns índices utilizados são o NDVI (Normalized Difference Vegetation Index), o NDWI (Normalized Difference Water Index) e o NDBI (Normalized Difference Built-Up Index) que realçam a presença, respectivamente, de áreas vegetadas, áreas úmidas e áreas urbanizadas (Lee et al., 2010; Moreira Filho \& Tavares Junior, 2016).

Com o uso das composições entre as diferentes bandas e da análise dos índices citados, é possível realizar a separação das classes de cobertura de uma determinada região (como uma bacia hidrográfica, município, estado, ou outras de acordo com o interesse do estudo) pela classificação digital de imagens. Esta classificação consiste em se agrupar pixels que possuem as mesmas características espectrais em diferentes bandas da imagem. 0 agrupamento pode ser supervisionado pelo usuário, baseado em seu conhecimento e com a identificação de amostras de áreas pertencentes a determinadas classes, ou a partir de algoritmos computacionais no qual o usuário somente informa 
o número de classes presentes na imagem. Ou seja, a classificação pode ser dividida em supervisionada ou não supervisionada (Centeno, 2009).

No campo dos estudos hidrológicos um método simples e amplamente utilizado é a curva-número (CN), um parâmetro de modelo chuva-vazão desenvolvido pelo órgão do Departamento de Agricultura dos Estados Unidos, Soil Conservation Service (SCS), que pode ser obtida a partir de informações de sensoriamento remoto com o cruzamento entre tipo de solo e a forma de ocupação do mesmo (Soares et al., 2017).

0 modelo empírico SCS-CN é baseado no balanço hídrico e nas hipóteses de que: a) a razão entre o escoamento superficial efetivo e o escoamento superficial potencial máximo é igual à razão entre a infiltração efetiva e a infiltração potencial máxima; e b) a abstração inicial (água retida na vegetação e em sulcos no solo, por exemplo) é uma fração da infiltração potencial máxima (Mishra \& Singh, 1999). A infiltração potencial máxima é dependente da CN, que por sua vez é obtida a partir das características da bacia (Mishra \& Singh, 1999).

O cálculo da CN consiste em separar os diversos solos em quatro tipos hidrológicos (A, B, C e D) de acordo com sua permeabilidade e potencial de escoamento. Os solos do tipo A são aqueles com maior capacidade de infiltração e, portanto, menor potencial de escoamento, como areias, e o tipo D os solos com baixa permeabilidade e alto potencial de gerar escoamento superficial, como argilas. A $\mathrm{CN}$ total de uma bacia hidrográfica é obtida pela média ponderada entre as diversas $\mathrm{CN}_{\mathrm{i}}$ parciais de cada polígono formado por um determinado tipo de solo e sua respectiva cobertura. Quanto maior o valor de CN, maior o fator de impermeabilização do solo e, consequentemente, maior a capacidade de escoamento superficial, ou seja, maior a capacidade da bacia de transformar chuva em vazão (United States Department of Agriculture, 1986).

Neste artigo, o avanço antrópico sobre a bacia hidrográfica do Rio Barigüi foi avaliado a partir da comparação da cobertura do solo nos anos de 1999 e 2019. Para tanto, foram obtidas imagens dos satélites da série Landsat, cena 220/78, nas datas de 02/09/1999 (Landsat 5) e 09/09/2019 (Landsat 8), no site do Serviço Geológico dos Estados Unidos (USGS) e o shape da bacia do Rio Barigüi no site do Instituto de Águas do Paraná (Instituto Água e Terra, 2000). As duas imagens foram obtidas para o mesmo mês em cada ano a fim de respeitar a sazonalidade que poderia influenciar nas áreas vegetadas e evitar erros devido à confusão na classificação de solo exposto e área agrícola, visto que da forma como foi realizada as culturas deverão estar no mesmo estágio de crescimento entre as imagens analisadas.

O sistema de projeção utilizado foi o mesmo do shape da bacia disponibilizado, SAD 69 UTM 22S. Para proceder a classificação da cobertura do solo da bacia, as bandas vermelha, verde, azul e as infravermelhas próxima e média foram empilhadas e, em seguida, realizado o corte da bacia a partir de shape do contorno. Em seguida, os índices NDVI, NDWI e NDBI foram calculados conforme as equações a seguir (Lee et al, 2010; Moreira Filho \& Tavares Junior, 2016):

$$
\begin{aligned}
& \text { NDVI }=\frac{\left(\rho_{\text {IRn }}-\rho_{R}\right)}{\left(\rho_{\text {IRn }}+\rho_{R}\right)} \\
& \text { NDWI }=\frac{\left(\rho_{\mathrm{G}}-\rho_{\text {IRn }}\right)}{\left(\rho_{\mathrm{G}}+\rho_{\mathrm{IRn}}\right)} \\
& \mathrm{NDBI}=\frac{\left(\rho_{\mathrm{IRm}}-\rho_{\mathrm{IRn}}\right)}{\left(\rho_{\mathrm{IRm}}+\rho_{\mathrm{IRn}}\right)}
\end{aligned}
$$

sendo $\rho_{\mathrm{IRn}}$ a refletância no infravermelho próximo, $\rho_{\mathrm{R}}$ no vermelho, $\rho_{\mathrm{G}}$ no verde e $\rho_{\mathrm{IRm}}$ no infravermelho médio (Lee et al, 2010; Moreira Filho \& Tavares Junior, 2016). Por tratarem-se de índices normalizados seus valores variam de -1 a +1 . No caso do NDVI valores positivos indicam presença de vegetação saudável e valores negativos de áreas urbanas, solo exposto ou vegetação com estresse hídrico; valores positivos de NDWI são relacionados a corpos hídricos, áreas úmidas e vegetação com maior umidade; no NDBI valores positivos indicam a presença de áreas edificadas e solo exposto (Teixeira et al., 2017; Karanam \& Babuneela, 2017).

Visando facilitar a classificação da cobertura do solo, as bandas originais do satélite foram novamente empilhadas junto às imagens geradas pelo cálculo dos índices radiométricos. Para identificação da classe de cada amostra utilizou-se as combinações de cor verdadeira (RGB), falsa cor infravermelho (infravermelho próximo, vermelho e verde) e falsa cor simulada (infravermelhos 
próximo e médio e vermelho), além dos índices NDVI, NDWI e NDBI e imagens dos anos analisados (1999 e 2019), quando possível, do Google Earth Pro e Street View (Google Earth Pro, 2020).

Desta forma, foi realizada a classificação supervisionada com a seleção de amostras que abrangessem todas as classes de cobertura observadas na região de interesse de forma homogênea, sendo possível a separação entre as seguintes classes: vegetação arbórea, vegetação rasteira, área agrícola, solo exposto (tanto urbano, quanto rural), área urbana e área industrial ou comercial de grande porte (caracterizados por galpões), água e áreas alagadiças.

Após a classificação, as imagens resultantes foram novamente comparadas às do Google Earth Pro (Google Earth Pro, 2020) e, caso necessário, correções foram realizadas a partir da seleção de novas amostras. Ainda, a fim de avaliar o desempenho da classificação, foi calculado o coeficiente Kappa (K), que leva em consideração a matriz de erros de classificação para avaliar o desempenho da classificação da imagem. Seu valor varia de 0 a 1, sendo que quanto mais perto de 1 , melhor é a classificação da imagem (Centeno, 2009). Os recortes, empilhamentos e classificação das imagens foram realizados nos softwares livres QGis e MultiSpec.

Um diagrama resumindo os passos da metodologia encontra-se representado na Figura 3.

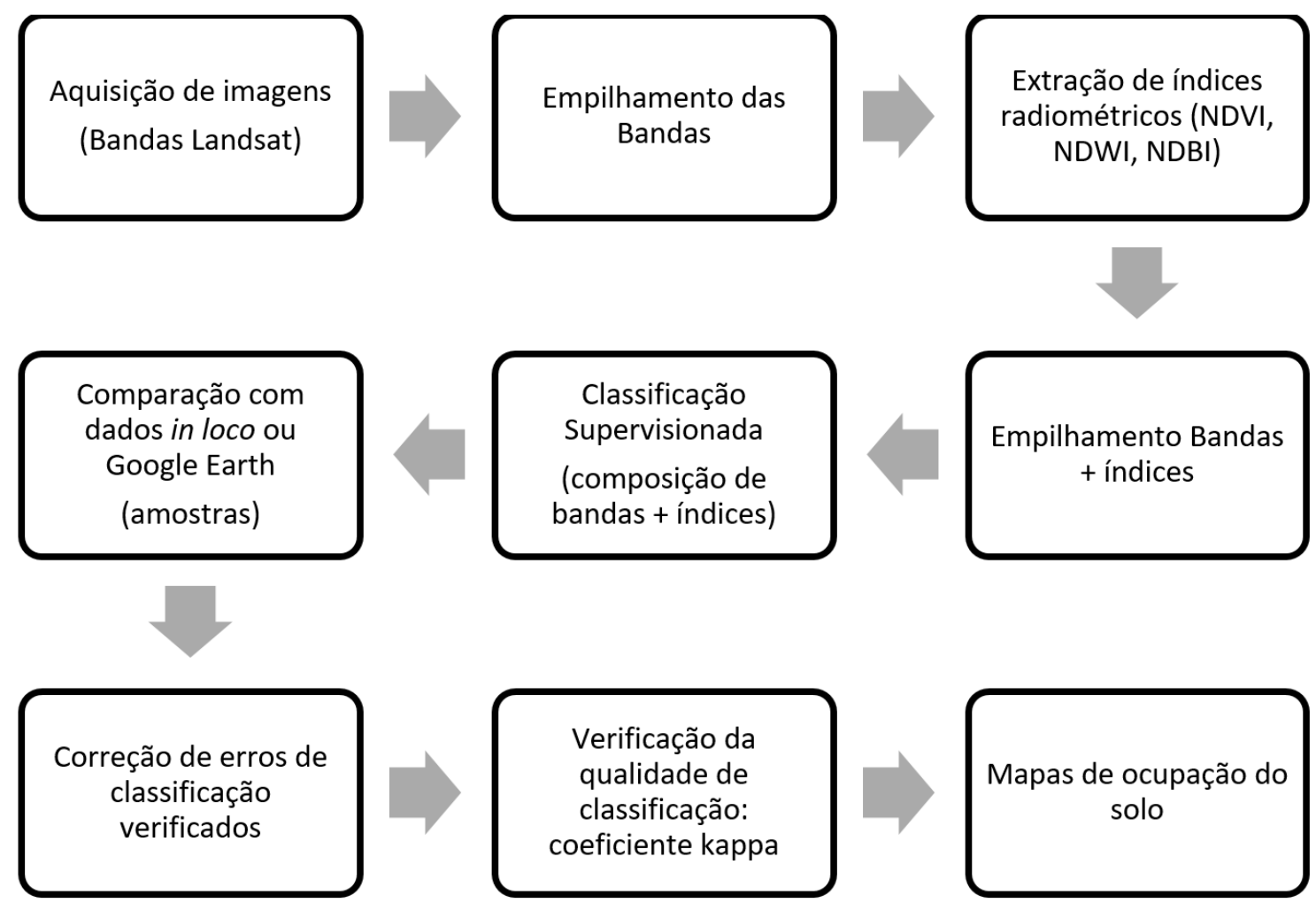

Figura 3 - Resumo Esquemático da Metodologia Aplicada

\section{Estimativa do valor de curva-número $\mathrm{CN}$}

O impacto da alteração da cobertura do solo no período de 20 anos sobre as características hidrológicas da bacia em estudo foi estimado através da curva-número (CN) calculada para os anos de 1999 e 2019 . Assim, além da cobertura foi necessário obter os tipos de solo da bacia a partir do recorte do mapa de Solos do Estado, disponível no Instituto de Terras, Cartografia e Geologia do Paraná (Instituto de Terras Cartografia e Geologia do Paraná, 2008).

Em seguida, os solos presentes na bacia do Barigüi foram enquadrados entre os tipos hidrológicos A, B, C e D, conforme classificação do Departamento de Agricultura dos Estados Unidos (United States Department of Agriculture, 1986), não tendo sido constatado nenhum solo do tipo B no interior da bacia hidrográfica. Ainda, nos mapas oficiais não há indicação dos tipos de solo presentes na parte central do município de Curitiba, sendo este local definido apenas como "Área Urbana". Assim, devido ao seu alto adensamento e forte impermeabilização do solo esta região foi classificada neste estudo como solo do tipo D para a composição da CN. 
Os tipos de solo presentes na bacia de acordo com as informações oficiais e suas respectivas classificações entre os tipos hidrológicos estão apresentados na Figura 4 e suas áreas resumidas na Tabela 1.

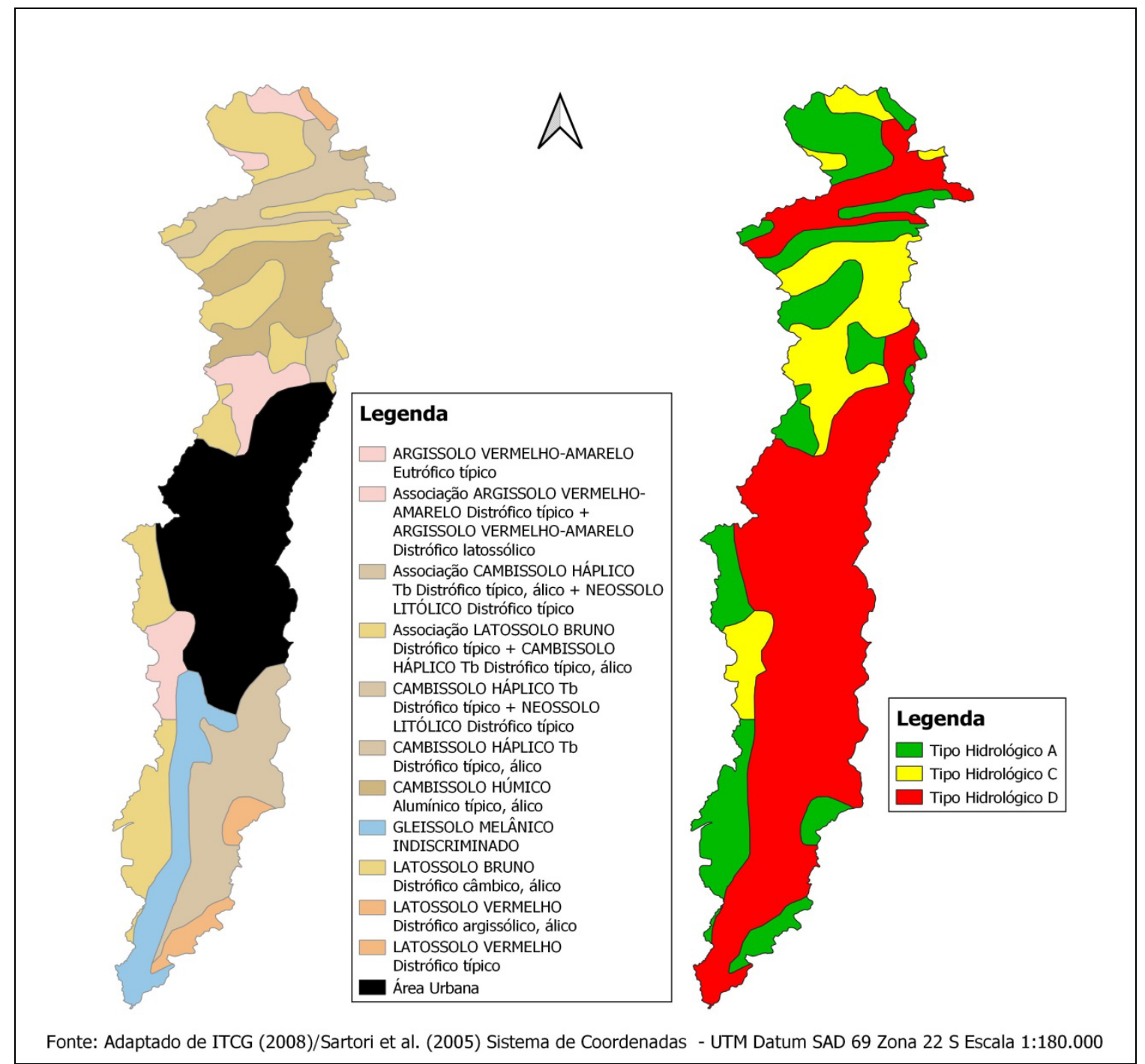

Figura 4 - Solos presentas na Bacia do Rio Barigüi (esquerda) e seus respectivos tipos hidrológicos de acordo com o método SCS-CN (direita)

Tabela 1 - Áreas dos tipos de solo

\begin{tabular}{c|c}
\hline Tipo de Solo & Área $\left(\mathbf{k m}^{\mathbf{2}}\right)$ \\
\hline A & 68 \\
\hline C & 44 \\
\hline D & 113 \\
\hline Área Urbana & 40 \\
\hline
\end{tabular}

Em seguida, a curva-número parcial $\left(\mathrm{CN}_{\mathrm{i}}\right)$ para cada combinação de cobertura $\mathrm{x}$ tipo hidrológico de solo foi obtida a partir da Technical Release 55 (TR-55), que apresenta procedimentos para cálculos hidrológicos em pequenas bacias, especialmente nos Estados Unidos, estando entre eles o modelo chuva-vazão CN-SCS (United States Department of Agriculture, 1986), e pela simplificação realizada para utilização de classificação realizada a partir de imagens de satélite na região em estudo disponível (estado do Paraná) em Soares et al. (2017), conforme Tabela 2. 
Tabela 2 - Valores de $\mathrm{CN}_{\mathrm{i}}$ utilizados para cada tipo de solo na área em estudo

\begin{tabular}{l|c|c|c|c}
\hline Uso/Tipo de Solo & A & B & C & D \\
\hline Vegetação Arbórea & 36 & 60 & 70 & 76 \\
\hline Vegetação Rasteira & 25 & 59 & 75 & 83 \\
\hline Área Agrícola & 62 & 71 & 78 & 81 \\
\hline Solo Exposto & 77 & 86 & 91 & 94 \\
\hline Área Urbana & 77 & 85 & 90 & 92 \\
\hline Área Comercial/Industrial & 81 & 88 & 91 & 93 \\
\hline Água & 100 & 100 & 100 & 100 \\
\hline Área Alagadiças & 100 & 100 & 100 & 100 \\
\hline
\end{tabular}

os valores de CN total para a bacia do Rio Barigüi nos anos de 1999 e 2019 foram calculados a partir da média ponderada das $\mathrm{CN}_{\mathrm{i}}$ parciais de cada polígono de tipo versus uso do solo.

\section{RESULTADOS E DISCUSSÃO}

\section{Evolução da cobertura do solo}

A Figura 5 demonstra a classificação da cobertura do solo realizada para os anos 1999 e 2019 com base nas características das bandas e índices radiométricos das imagens de satélite.

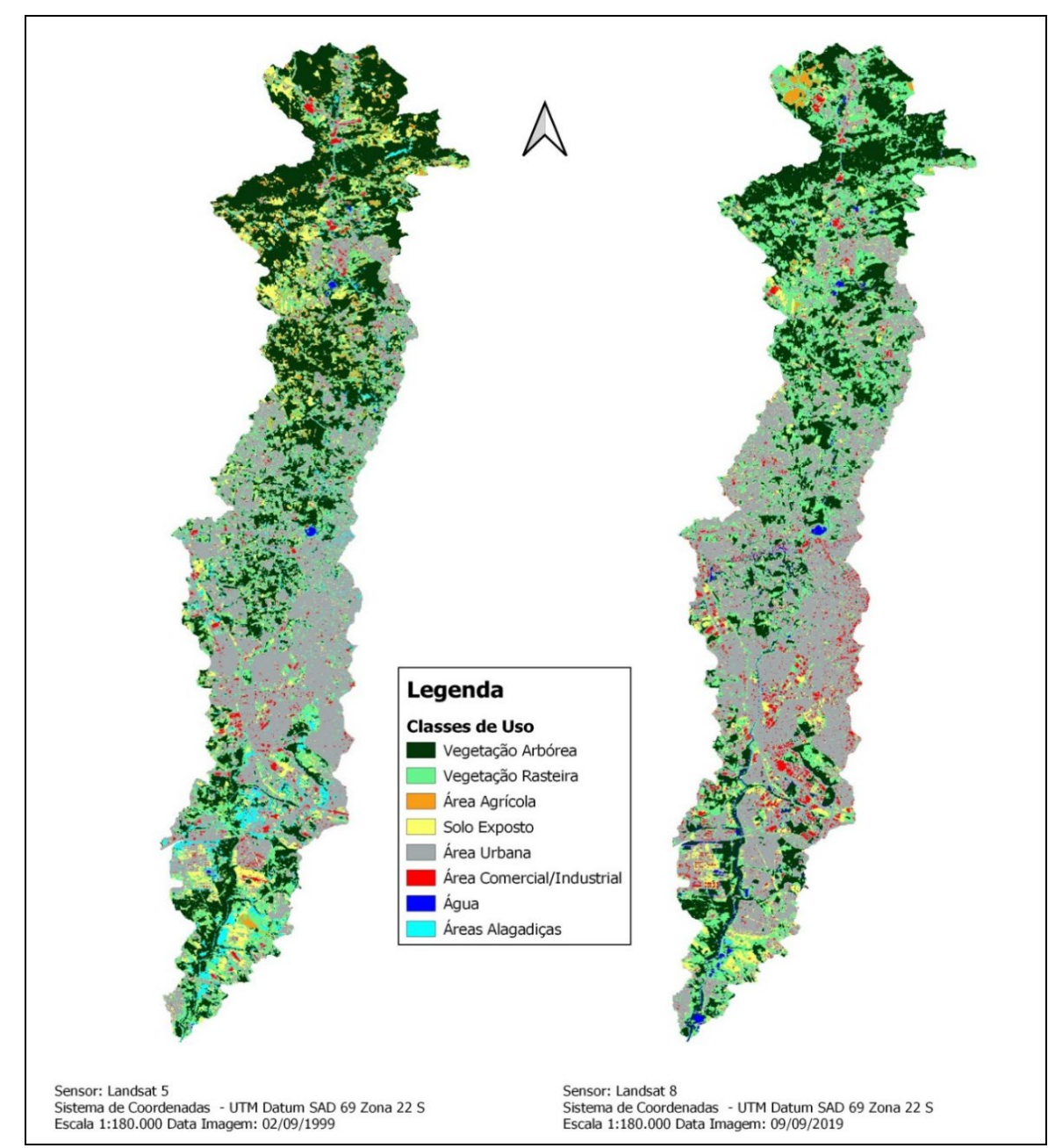

Figura 5 - Classificação realizada da cobertura do solo na Bacia do Rio Barigüi em 1999 (esquerda) e 2019 (direita) 
Um resumo com as diferenças de cobertura do solo entre os dois períodos analisados encontrase na Tabela 3.

Tabela 3 - Áreas de cada cobertura de solo na Bacia do Rio Barigüi para os anos de 1999 e 2019

\begin{tabular}{|c|c|c|c|}
\hline Classe & Área em $1999\left(\mathrm{~km}^{2}\right)$ & Área em $2019\left(\mathrm{~km}^{2}\right)$ & Evolução \\
\hline Vegetação Arbórea & 84,4 & 62,6 & $-26 \%$ \\
\hline Vegetação Rasteira & 44,4 & 64,2 & $44 \%$ \\
\hline Área Agrícola & 7,3 & 1,5 & $-79 \%$ \\
\hline Solo Exposto & 20,2 & 15,0 & $-26 \%$ \\
\hline Área Urbana & 90,5 & 107,6 & $19 \%$ \\
\hline Área Comercial/Industrial & 5,7 & 10,1 & $76 \%$ \\
\hline Água & 1,4 & 3,8 & $176 \%$ \\
\hline Área Alagadiças & 11,0 & 0,1 & $-99 \%$ \\
\hline Total & 265 & 265 & \\
\hline
\end{tabular}

O desempenho da classificação das imagens foi considerado excelente, visto que o índice kappa foi $95 \%$ para as duas imagens classificadas. No entanto, é preciso destacar que não foi realizada qualquer correção atmosférica ou topográfica nas imagens utilizadas.

A partir da classificação das imagens verifica-se que a área urbana sofreu uma expansão de quase $20 \%$ e a área comercial/industrial de $76 \%$ ao longo dos 20 anos estudados. Isto representa um aumento de solo impermeabilizado de mais de $21 \mathrm{~km}^{2}$ (em torno de 8,1\% da área total da bacia), o que gera impacto direto sobre o escoamento superficial.

Uma alteração que se destaca nas imagens da Figura 4 e na Tabela 3 é a expansão da área com vegetação rasteira. Ao comparar as imagens verifica-se que tal expansão ocorreu tanto pela redução da vegetação arbórea, quanto das áreas com solo exposto, possivelmente pela utilização destes espaços como pasto, principalmente ao norte da bacia. Assim, um impacto direto do avanço antrópico, tanto rural, como urbano, foi a redução de áreas florestadas, uma área de aproximadamente $22 \mathrm{~km}^{2}$ (cerca de 8,2\% da área total).

Comparando-se as imagens classificadas verificou-se a redução de áreas alagadiças (de 11 para $0,1 \mathrm{~km}^{2}$ ) devido ao seu aterro para a expansão da área urbana, gerando pequenas lagoas para drenagem dos terrenos, principalmente na região sul da bacia (aumento de área com águas superficiais de 1,4 para 3,8 km²). As áreas agrícolas apresentaram também expressiva redução, quase $6 \mathrm{~km}^{2}$.

Porém, mesmo as imagens sendo do mesmo mês para garantir que as culturas estejam no mesmo nível de desenvolvimento nos dois anos em análise, devido ao amplo período de tempo em estudo uma alteração nas espécies cultivadas na bacia pode ter ocorrido, não sendo possível afirmar, com base na resolução espacial das imagens, qual o real grau de redução da área agrícola, pois estas podem estar representadas nas duas imagens por áreas de solo exposto ou coberto por vegetação rasteira.

\section{Mudança no valor da curva-número}

As contribuições de cada uso do solo para os tipos de solo no valor total do CN são apresentadas na Tabela 4. Geralmente, a redução de áreas vegetadas e a expansão urbana levam a um aumento no parâmetro $\mathrm{CN}$ devido à maior impermeabilização do solo da bacia, e, consequente, maior escoamento superficial. Isso se observa, por exemplo, no estudo de Soares et al. (2017) no qual a CN teve um aumento de forma generalizada no período analisado, devido à expansão urbana, principalmente. No entanto, no presente estudo de caso, apesar do nítido aumento da área urbana e redução das regiões arborizadas ocorridas na bacia, não houve alteração significativa no valor total de CN, sendo 76,9 em 1999 contra 76,7 em 2019, ocorrendo até mesmo uma pequena redução em 2019 (cerca de 0,28\%), o que contraria a expectativa inicial. 
Tabela $4-\mathrm{CN}_{\mathrm{i}}$ utilizados para cada tipo de solo e seus valores totais para a bacia nos anos de 1999 e 2019

\begin{tabular}{|c|c|c|c|c|c|c|c|c|}
\hline \multirow{2}{*}{ Uso do solo } & \multicolumn{4}{|c|}{1999} & \multicolumn{4}{|c|}{2019} \\
\hline & $\mathbf{C N}_{\mathrm{A}}$ & $\mathrm{CN}_{\mathrm{C}}$ & $\mathrm{CN}_{\mathbf{D}}$ & CNuso & $\mathrm{CN}_{\mathrm{A}}$ & $\mathrm{CN}_{\mathrm{C}}$ & $\mathbf{C N}_{\mathbf{D}}$ & CNuso \\
\hline Vegetação Arbórea & 3,3 & 5,2 & 11,6 & 20,1 & 2,3 & 4,0 & 8,8 & 15,1 \\
\hline Vegetação Rasteira & 1,1 & 2,0 & 8,1 & 11,2 & 1,9 & 3,8 & 9,6 & 15,3 \\
\hline Área Agrícola & 0,6 & 0,7 & 0,7 & 2,0 & 0,3 & 0,1 & 0,0 & 0,4 \\
\hline Solo Exposto & 2,2 & 1,2 & 3,3 & 6,7 & 1,6 & 0,6 & 2,7 & 4,9 \\
\hline Área Urbana & 5,0 & 3,3 & 22,1 & 30,3 & 5,9 & 4,0 & 26,2 & 36,1 \\
\hline Área Comercial/Industrial & 0,5 & 0,3 & 1,1 & 1,9 & 0,9 & 0,3 & 2,3 & 3,4 \\
\hline Água & 0,1 & 0,1 & 0,3 & 0,5 & 0,3 & 0,1 & 1,0 & 1,4 \\
\hline Área Alagadiças & 1,0 & 0,3 & 2,9 & 4,2 & 0,0 & 0,0 & 0,0 & 0,0 \\
\hline $\mathrm{CN}_{\text {Total }}$ & 13,9 & 12,9 & 50,1 & 76,9 & 13,3 & 12,9 & 50,5 & 76,7 \\
\hline
\end{tabular}

Para compreender tal aparente contradição, inicialmente foram avaliados os tipos de solo da bacia ficando constatado que mais de $40 \%$ da área possui solos do tipo D, os quais possuem baixa capacidade de infiltração e alta geração de escoamento superficial. Desta forma, as alterações de cobertura sobre este tipo de solo não geram impactos tão relevantes no $\mathrm{CN}$ quanto àquelas ocorridas em solos mais permeáveis.

Além disso, foram avaliadas e comparadas as $\mathrm{CN}_{\mathrm{i}}$ parciais de cada tipo de solo e de cobertura separadamente e em conjunto. Com isso constatou-se que aquelas coberturas que geram escoamento superficial e infiltração $\left(\mathrm{CN}_{\mathrm{i}}<100\right.$ : vegetação arbórea e rasteira, solo exposto, áreas urbana e comercial/industrial) tiveram uma elevação no CN ponderado entre essas classes de 72,3 para 75,2, enquanto as áreas alagadiças e águas superficiais $\left(\mathrm{CN}_{\mathrm{i}}=100\right)$ produziram uma redução do $\mathrm{CN}$ ponderado de 4,7 para 1,5. Assim, ao calcular o valor final da CN houve uma compensação da elevação do seu valor pela maior impermeabilização da área devido à drenagem e aterramento das áreas alagadiças. De forma semelhante, Soares et al. (2017) apesar de verificarem um aumento generalizado na CN das sub-bacias do Ivaí, também observaram alguns pixels nos quais houve redução ou manutenção do valor de CN, compensando, de certa forma, o acréscimo de impermeabilização de outros pixels no cálculo da CN por sub-bacia.

Desta forma, apesar de verificar a expansão antrópica na bacia do Rio Barigüi no período em análise constatada através da classificação da cobertura do solo realizada a partir de imagens de satélite, com notória redução de áreas arborizadas e ampliação de regiões urbanizadas, não houve uma alteração relevante do valor final do CN calculado para a bacia como um todo, principalmente por esta ocorrer em áreas anteriormente alagadiças $\left(\mathrm{CN}_{\mathrm{i}}=100\right)$ que foram aterradas $\left(\mathrm{CN}_{\mathrm{i}}<100\right)$. Entretanto, houve modificação nos valores parciais das $\mathrm{CN}_{\mathrm{i}}$ o que indica possível alteração nas características hidrológicas da bacia quando esta é analisada por sub-bacias.

\section{CONSIDERAÇÕES FINAIS}

A cobertura do solo de uma bacia hidrográfica exerce forte influência sobre seus recursos hídricos como, por exemplo, sobre suas características hidrológicas. A utilização de Sistemas de Informação Geográfica (SIG) podem reduzir custos na gestão de recursos hídricos por permitir que esta seja realizada de forma remota, ao menos em parte, e visto que imagens de satélite, como as da série Landsat, podem ser obtidas sem custos e manipuladas a partir de softwares livres.

Comparando-se a cobertura do solo na bacia hidrográfica do Rio Barigüi entre os anos de 1999 e 2019 a partir da classificação das imagens Landsat verificou-se que o avanço antrópico gerou modificações na paisagem da bacia ao longo do período analisado, com provável alteração de seu comportamento hidrológico. No entanto, após o cálculo da curva-número não foi constatada variação relevante do seu valor quando considerada a bacia em sua totalidade, inclusive com uma pequena redução da CN obtida para o ano 2019 em comparação a 1999.

Tal fato é devido em parte ao tipo hidrológico predominante na bacia, tipo D, o qual possui baixa permeabilidade independente de sua ocupação, estando presente principalmente nos locais onde houve expansão da área urbana (região central e sul da bacia). Além disso, a redução das áreas alagadiças por aterro compensou a impermeabilização de outras regiões da bacia que tiveram redução de áreas arborizadas e aumento da área urbana. 
Assim, a divisão da área total em sub-bacias, com a determinação da CN e das características hidrológicas por seção, pode ser fundamental para garantir a avaliação mais acurada do escoamento superficial da bacia do rio Barigüi, mesmo possuindo tamanho compatível ao método CN-SCS. Isto porque, os valores de $\mathrm{CN}_{\mathrm{i}}$ de cada tipo de cobertura tiveram relevante variação, indicando alterações no escoamento superficial em diferentes trechos da bacia.

No entanto, tal fenômeno deixou de ser descrito pelo valor total da CN na bacia, o qual permaneceu praticamente inalterado no período analisado, o que ocorreu principalmente devido a uma compensação de permeabilidade do solo em regiões diferentes da bacia, e não realmente à manutenção das características hidrológicas na bacia ao longo destes 20 anos.

Desta forma, sugere-se que seja realizada a avaliação do método curva-número em sub-bacias menores, juntamente a dados de forma, como declividade e altimetria, para permitir a avaliação pelos gestores de recursos hídricos de quais áreas da bacia hidrográfica são mais sensíveis a alagamentos e enchentes, permitindo uma gestão de recursos hídricos mais eficiente e econômica.

\section{AGRADECIMENTOS}

O presente trabalho foi realizado com apoio da Coordenação de Aperfeiçoamento de Pessoal de Nível Superior - Brasil (CAPES) - Código de Financiamento 001 e do Conselho Nacional de Pesquisa CNPq.

\section{REFERÊNCIAS}

AMBDATA - INPE. (2019) Variáveis Ambientais para Modelagem de distribuição de espécies. Recuperado em 09 de fevereiro de 2020, de http://www.dpi.inpe.br/Ambdata/unidades_administrativas.php.

Bartlett, M. S., Parolari, A. J., McDonnell, J. J., \& Porporato, A. (2016). Beyond the SCS-CN method: A theoretical framework for spatially lumped rainfall-runoff response. Water Resources Research, 52(6), 4608-4627.

Beck, N. G., Conley, G., Kanner, L., \& Mathias, M. (2017). An urban runoff model designed to inform stormwater management decisions. Journal of Environmental Management, 193, 257-269.

Centeno, J. A. S. (2009). Sensoriamento remoto e processos de imagens digitais. Curitiba: Editora UFPR.

Froehner, S., \& Martins, R. F. (2008). Avaliação da composição química de sedimentos do Rio Barigüi na região metropolitana de Curitiba. Quimica Nova, 31(8), 2020-2026.

Gaglio, M., Aschonitis, V., Pieretti, L., Santos, L., Gissi, E., Castaldelli, G., \& Fano, E. A. (2019). Modelling past, present and future Ecosystem Services supply in a protected floodplain under land use and climate changes. Ecological Modelling, 403, 23-34.

Gonçalves, A. K., da Silveira, G. R. P., De Barros, Z. X., \& Campos, S. (2015). Métodos de classificação supervisionada de imagens de satélite aplicadas no mapeamento do uso do solo na bacia hidrográfica do Ribeirão Santo Antônio, São Manuel/SP. Geosaberes. Revista de Estudos Geoeducacionais, 6(1), 52-62.

Google Earth Pro. (2020). Recuperado em 15 de janeiro de 2020, de https://www.google.com/intl/ptBR/earth/desktop/.

Instituto Água e Terra. Superintendência de Desenvolvimento de Recursos Hídricos e Saneamento Ambiental. (2000). Mapas e Dados Espaciais. Recuperado em 12 de janeiro de 2020, de http://www.iat.pr.gov.br/Pagina/Mapas-e-Dados-Espaciais

Instituto Ambiental do Paraná - IAP. (2018). Monitoramento da qualidade das águas dos rios na Bacia do Alto Iguaçu, na Região Metropolitana de Curitiba: no período de 2010 a 2018. Recuperado em 11 de janeiro de 2020, de http://www.iap.pr.gov.br/arquivos/File/Qualidade_das_aguas/Relatorio_Alto_Iguacu_2018.pdf

Instituto das Águas do Paraná - AGUASPARANÁ. (2018). Mapas e Dados Espaciais. Recuperado em 08 de maio, de 2018, de http://www.aguasparana.pr.gov.br/pagina-78.html.

Instituto de Terras Cartografia e Geologia do Paraná - ITCG. (2008) Mapa de Solos. Recuperado 30 de janeiro de 2020, de http://www.itcg.pr.gov.br/modules/faq/category.php?categoryid=9\#.

Instituto de Terras Cartografia e Geologia do Paraná - ITCG. (2019) Divisão Político-Administrativa. Recuperado em 09 de janeiro de 2020, de http://www.itcg.pr.gov.br/modules/faq/category.php?categoryid=8\#.

Karanam, H. K., \& Babuneela, V. (2017). Study of normalized difference built-up (NDBI) index in automatically mapping urban areas from Landsat TM imagery. International Journal of Engineering Science, 6, 239-248. 
Kayet, N., Pathak, K., Chakrabarty, A., \& Sahoo, S. (2018). Evaluation of soil loss estimation using the RUSLE model and SCS-CN method in hillslope mining areas. International Soil and Water Conservation Research, 6(1), 31-42.

Lee, J. I. N. A., Lee, S. S., \& Chi, K. H. (2010). Development of an urban classification method using a built-up index. In Sixth WSEAS International Conference on Remote Sensing, (pp. 39-43). Japan: Iwate Prefectural University.

Machado, K. S., Figueira, R. C., Côcco, L. C., Froehner, S., Fernandes, C. V., \& Ferreira, P. A. (2014). Sedimentary record of PAHs in the Barigui River and its relation to the socioeconomic development of Curitiba, Brazil. The Science of the Total Environment, 482, 42-52.

Mendes, C. A. B., \& Cirilo, J. A. (2013). Geoprocessamento em recursos hídricos: princípios, integração e aplicação (2. ed.). ABRH.

Mishra, S. K., \& Singh, V. P. (1999). Another look at SCS-CN method. Journal of Hydrologic Engineering, 4(3), 257264.

Mishra, S. K., Singh, V. P., Sansalone, J. J., \& Aravamuthan, V. (2003). A modified SCS-CN method: characterization and testing. Water Resources Management, 17(1), 37-68.

Moreira Filho, J. C. C. \& Tavares Junior, J. R. (2016). Avaliação da precisão temática de composições de NDBI, NDVI, NDWI. Revista Brasileira de Geomática, 4(1).

Nachshon, U., Netzer, L., \& Livshitz, Y. (2016). Land cover properties and rain water harvesting in urban environments. Sustainable cities and society, 27, 398-406.

Paule-Mercado, M. C. A., Salim, I., Lee, B. Y., Memon, S., Sajjad, R. U., Sukhbaatar, C., \& Lee, C. H. (2018). Monitoring and quantification of stormwater runoff from mixed land use and land cover catchment in response to land development. Ecological Indicators, 93, 1112-1125.

Ramakrishnan, D., Bandyopadhyay, A., \& Kusuma, K. N. (2009). SCS-CN and GIS-based approach for identifying potential water harvesting sites in the Kali Watershed, Mahi River Basin, India. Journal of Earth System Science, 118(4), 355-368.

Soares, M. R. G. D. J., Fiori, C. O., Silveira, C. T. D., \& Kaviski, E. (2017). Eficiência do método Curve Number de retenção de águas pluviais. Mercator (Fortaleza), 16.

Superintendência de Desenvolvimento de Recursos Hídricos e Saneamento Ambiental - SUDERHSA. (2002). Plano Diretor de Drenagem da Bacia do Alto Iguaçu na Região Metropolitana de Curitiba. Recuperado em 12 de janeiro de 2020, de http://www.aguasparana.pr.gov.br/modules/conteudo/conteudo.php?conteudo=265.

Teixeira, A. M. A.; Candeias, A. L. B.; Tavares Junior, J. R. (2017). Comparação dos índices de vegetação para imagens TM/Landsat 5, com correção e sem correção atmosférica. Revista Brasileira de Geomática, 5(4), 524-542.

Tong, S. T., \& Chen, W. (2002). Modeling the relationship between land use and surface water quality. Journal of Environmental Management, 66(4), 377-393.

United States Department of Agriculture - USDA. (1986). Technical release 55: Urban hydrology for small watersheds.

Vale, J. R. B., da Costa, J. A., dos Santos, J. F., da Silva, E. L. S., \& Favacho, A. T. (2018). Análise comparativa de métodos de classificação supervisionada aplicada ao mapeamento da cobertura do solo no município de Medicilândia, Pará. InterEspaço. Revista de Geografia e Interdisciplinaridade, 4(13), 26-44.

Walega, A., Amatya, D. M., Caldwell, P., Marion, D., \& Panda, S. (2020). Assessment of storm direct runoff and peak flow rates using improved SCS-CN models for selected forested watersheds in the Southeastern United States. Journal of Hydrology: Regional Studies, 27, 100645.

\section{Contribuições dos autores:}

Juliana Leithold: Compilação e análise de dados, produção de tabelas e figuras, redação, formatação.

Marcio Augusto Reolon Schmidt: Correções e revisão de conceitos e texto

Cristóvão Vicente Scapulatempo Fernandes: Orientação e revisão de texto 